
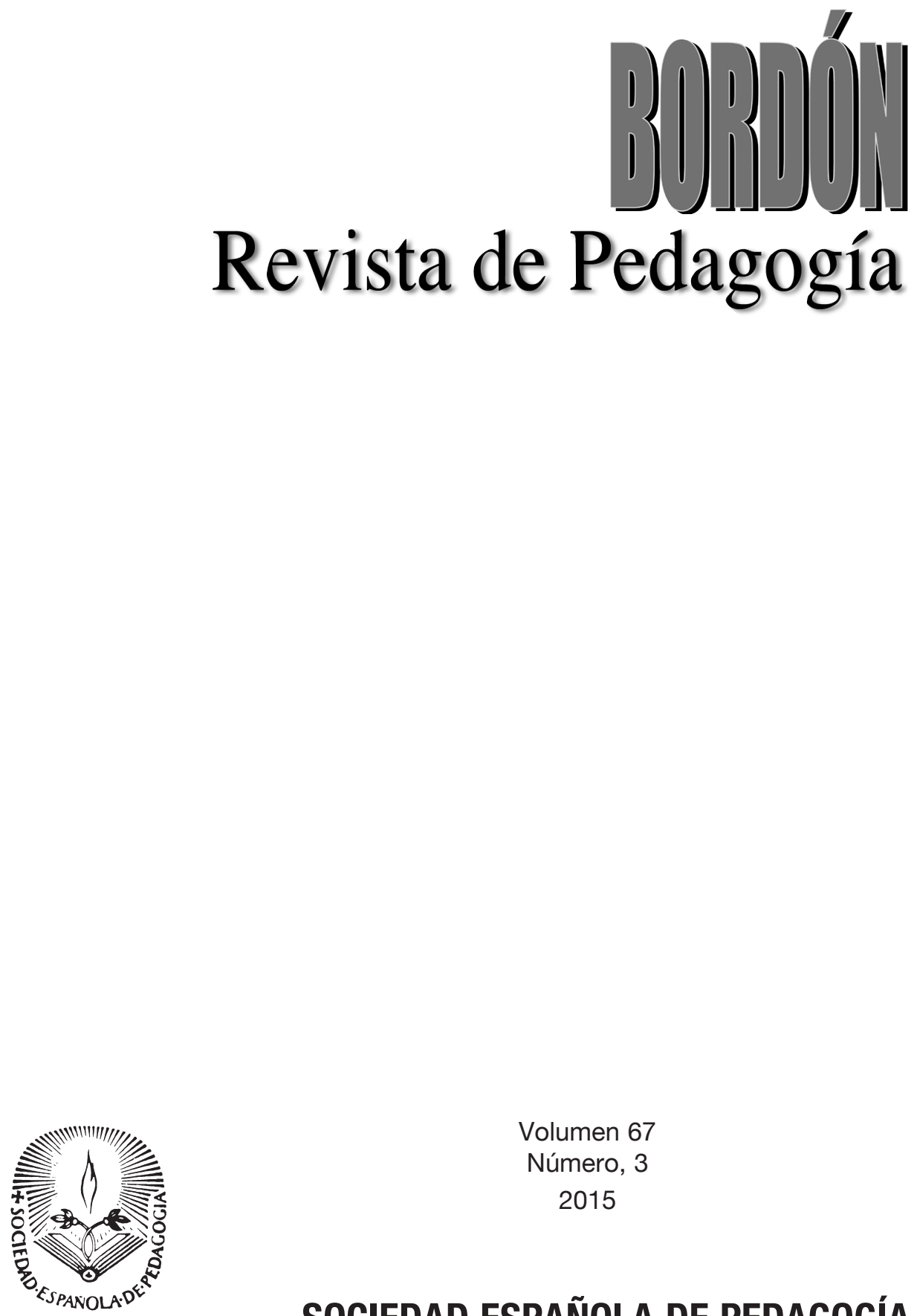

Volumen 67

Número, 3

2015

SOCIEDAD ESPAÑOLA DE PEDAGOGÍA 


\title{
PATRONES DIFERERCIALES ENTRE PADRES Y PROFESORADO En LA DETECCIÓn DE TDAH
}

\section{Differential patterns between parents and teachers in detecting ADHD}

\author{
PURIFICACIÓN SÁNCHEZ-DELGADO \\ Universitat de València \\ ANA ROSA BODOQUE OSMA \\ Universidad de Castila-La Mancha \\ JESÚS MIGUEL JORNET MELIÁ \\ Universitat de València
}

D01: 10.13042/Bordon.2015.67308

Fecha de recepción: 16/06/2014 - Fecha de aceptación: 20/10/2014

Autor de contacto / Corresponding Author: Purificación Sánchez-Delgado. E-mail: purificación.sanchez@uv.es

Fecha de publicación online: 18/05/2015

INTRODUCCIÓN. El diagnóstico del Trastorno por Déficit de Atención con Hiperactividad (TDAH) se ha ido incrementando en los últimos años (CDC, 2014; UNESCO, 2011: 14). Los criterios sobre el TDAH se han ido aclarando entre la comunidad científica. Sin embargo, se trata de un trastorno complejo que requiere la participación en el diagnóstico de un equipo multidisciplinar. MÉTODO. En este estudio se analiza, con una muestra $(\mathrm{N}=331)$ de alumnado de 6 a 9 años de Cuenca, la existencia de patrones diferenciales entre las dos valoraciones, mediante las Escalas EMA-D.D.A. (García-Pérez y Magaz, 2006). Se utilizan diversas aproximaciones de contraste de hipótesis, así como se valora la consistencia interna de las escalas. RESULTADOS. Las diferencias que se observan apuntan al influjo diferencial del contexto observacional y es coincidente con los resultados obtenidos en otros estudios. DISCUSIÓN. En el diagnóstico se requiere de una detección escolar, y una evaluación clínica y otra neurológica. En la detección escolar se utilizan escalas de evaluación comportamental, a las que responden padres y profesorado. El establecimiento del diagnóstico requiere que ambas valoraciones sean positivas, es decir, coincidentes en la detección de condición TDAH. Los estudios realizados muestran que existen diferencias entre ambas valoraciones hecho que analizaremos en la interpretación y conclusiones del presente trabajo.

Palabras clave: TDAH, Diagnóstico, Detección escolar, Análisis diferencial, Valoración de padres y profesorado. 


\section{Introducción}

En la iniciación del proceso de escolarización se espera que el niño cumpla las normas de disciplina y permanezca quieto durante largos periodos de tiempo, mientras desarrolla habilidades cognitivas y comportamentales. La falta de las habilidades requeridas, donde se pide al niño una atención y un comportamiento adecuados, hace que en numerosas ocasiones se confunda ese patrón inmaduro con manifestaciones de TDA con o sin hiperactividad. Este hecho condujo a que en los últimos años se detectase un aumento en el número de dictámenes dirigidos hacia el TDA-H, donde, de forma genérica, se incluyen alumnos cuyo diagnóstico no está claro.

Actualmente, nuestra sociedad sigue empleando el término "niño hiperactivo" como síntoma de un trastorno, indicio de posible TDAH, hecho que lleva a confusión a muchos padres y maestros. Cuando los niños no son bien diagnosticados pueden recibir tratamientos que les perjudiquen (Morrow, Wright, Maclure, Tailor y Dormuth, 2012). Por ello, es de suma importancia que aclaremos la diferencia entre niños inquietos, ansiosos o inadaptados emocional y socialmente de aquellos que presentan un comportamiento y funcionamiento cognitivo que puede llegar a ser patológico en el caso de que exista una elevada intensidad de una sintomatología, pudiendo generar problemas de adaptación para poder ofrecerles una atención educativa y social acorde a sus necesidades (Orjales, 2003).

La identificación de este tipo de niños o niñas nace inicialmente en el seno de la familia o en el de la escuela. Por ello, buena parte de las escalas que se utilizan para el diagnóstico implican la valoración del comportamiento de los niños por parte del profesorado y de los padres. Sin embargo, existen evidencias acerca de que ambas valoraciones no son concurrentes sino en todo caso complementarias, pues el tipo de comportamientos que pueden darse en ambos ambientes (escolar y sociofamiliar) no son necesariamente los mismos, ni tampoco la percepción que puedan tener al respecto padres y profesores. Su detección y diagnóstico es compleja pues necesita de una detección que puede ser escolar-familiar o clínica, pero el diagnóstico debe llevarlo a cabo un profesional clínico especializado.

El estudio que aquí presentamos forma parte de una investigación más amplia realizada en Cuenca, dirigida a estudiar las claves evaluativas que nos permitan mejorar los patrones diagnósticos de esta casuística.

En este artículo presentamos una breve revisión conceptual de la definición de TDAH, unas reflexiones acerca del proceso de evaluación/ diagnóstico para su detección escolar y un estudio diferencial entre los patrones de valoración que presentan padres y profesores a partir de las Escalas Magallanes de Detección de Déficit de Atención -EMA-D.D.A (García-Pérez y Magaz, 2006) en niños de entre 6 y 9 años de edad.

\section{Algunas notas respecto al concepto de TDA-H}

El trastorno que hoy en día conocemos como Trastorno por Déficit de Atención con Hiperactividad (TDAH) ha tenido múltiples nombres y ha sido determinado como alteración, trastorno, síntoma, síndrome y/o reacción. Esta variedad de términos refleja la dificultad para establecer un único concepto del mismo. Las primeras referencias acerca de esta problemática (aunque no identificada con esta denominación) se remontan al siglo XVIII. Sin embargo, no haremos una revisión detallada de su evolución, dado que excedería con mucho el espacio disponible, y nos centraremos en las características que se le reconocen al TDAH en la actualidad. En el cuadro 1 se recoge una síntesis histórica de la evolución del concepto hasta el DSM-IV, en el que por primera vez se distinguen tres subtipos: 
hiperactivo-impulsivo, hiperactivo-inatento y combinado. La revisión del manual dio como resultado el DSM-IV-TR (APA, 2002), en el que se definieron mejor los criterios de evaluación, así como la descripción de las diferentes categorías diagnósticas al recoger una información más detallada sobre los propios criterios diagnósticos, en la que se describían aspectos clínicos determinantes sobre los trastornos (Sandin, 2013).

\section{CuAdro 1. Síntesis histórica de la evolución del concepto de hiperactividad hasta el de TDA-H}

\begin{tabular}{|c|c|c|}
\hline Año & Autor & Referencia \\
\hline 1798 & Alexander Crinchton & Inquietud mental \\
\hline 1846 & Hoffman & El intranquilo \\
\hline 1867 & Maudsley & Perturbaciones conductuales \\
\hline 1876 & Ferrier & "Defecto de una función motor-inhibitoria" del lóbulo frontal \\
\hline 1877 & Ireland & Perturbaciones conductuales. \\
\hline 1892 & Cloustond & Estados de defectuosa inhibición asociados al exceso de actividad \\
\hline 1892 & Tuke & Síntoma de enfermedad impulsiva \\
\hline 1892 & Maudsley & Hiperactividad síntoma central del trastorno "niños inestables" \\
\hline 1897 & Bourneville & Inquietud psicomotora, inatención, indisciplina, desobediencia \\
\hline 1899 & Clouston & Perturbaciones conductuales. Hiperexcitabilidad \\
\hline 1901 & Denoor & Corea mental \\
\hline 1902 & Still & Defecto del control moral \\
\hline 1908 & Tredgold & Enfermedad neuropática \\
\hline 1909 & Dupré & Manifestación de un desequilibrio motor congénito \\
\hline 1901 & Demoor & Corea mental \\
\hline 1905 & Boncour & Escolar inestable \\
\hline 1913 & Durot & Pionero en diferenciar síntoma y síndrome \\
\hline 1914 & Heuyer & La hiperactividad como síndrome \\
\hline 1922 & Hoffman & Secuelas de la encefalitis letárgica \\
\hline 1923 & Vermeylen & Pionero en introducir variables de tipo neurocognitivo \\
\hline 1925 & Wallon & Alteración de la subcortezaintegradora \\
\hline 1930 & Kramer y Pollnow & Trastorno hipercinético \\
\hline 1934 & Kahn y Cohen & Síndrome de impulsividad orgánica \\
\hline 1936 & Blau & Lesiones en el lóbulo frontal \\
\hline 1938 & Levin & Lesiones en el lóbulo frontal \\
\hline 1947 & Strauss y Lehtinen & Síndrome de daño cerebral infantil \\
\hline 1956 & Hoff & Trastorno hipercinético \\
\hline 1957 & Laufer, Denhoff y Solomons & Déficit en el área talámica del SNC \\
\hline 1960 & Chess & Trastorno hipercinético \\
\hline 1968 & DSM-II & Reacción hipercinética de la infancia \\
\hline 1972 & Douglas & Trastorno por déficit de atención con y sin hiperactividad \\
\hline
\end{tabular}


CuAdro 1. Síntesis histórica de la evolución del concepto de hiperactividad hasta el de TDA-H (cont.)

\begin{tabular}{lll} 
Año & Autor & Referencia \\
\hline 1962 & Clemens y Peters & Daño cerebral mínimo \\
\hline 1978 & CIE-9 & Trastorno hipercinético de la infancia \\
\hline 1980 & DSM-III & Trastorno por déficit de atención con y sin hiperactividad \\
\hline 1987 & DSM-III-TR & Déficit de atención con hiperactividad \\
\hline 1992 & CIE-10 & Trastorno de la actividad y de la atención (en trastornos hipercinéticos) \\
\hline $1994 / 2000$ & DSM-IV y DSM-IV-TR & $\begin{array}{l}\text { El TDAH. Tres subtipos: hiperactivo-impulsivo, hiperactivo-inatento y } \\
\text { combinado }\end{array}$
\end{tabular}

Fuente: adaptado de Navarro González y García-Villamisar, 2010.

Los criterios normalizados para determinar el diagnóstico del TDAH y del subtipo se establecen mediante unos estándares clínicos, atendiéndose a la presencia/ausencia de unos síntomas y de su grado de intensidad, en nuestro caso hemos utilizado los establecidos en el DSM-IV-TR (APA, 2002). Se recomienda realizar el diagnóstico a partir de los seis años y debe constar de una evaluación psicológica, pedagógica y médica, que descarte que las manifestaciones no son debidas a otras causas. Estos mismos criterios están ahora recogidos en el DSM-5 (AAP, 2013) aunque con pequeños cambios y son coincidentes en su mayor parte con los de la CIE-10 (OMS, 1995), que en breve sacará su undécima edición. Casualmente las últimas versiones de ambos manuales son las más próximas entre sí, en cuanto a criterios y nomenclatura se refiere. En el cuadro 2 se recogen los criterios diagnósticos del TDAH en diferentes versiones del DSM y la CIE-10 de forma que nos permite observar los cambios que se han producido a lo largo de las distintas ediciones.

CuAdro 2. Comparación de los criterios diagnósticos del TDAH en diferentes versiones del DSM y la CIE-10

$\begin{array}{llll}\text { DSM-III } & \text { DSM-III-TR } & \begin{array}{l}\text { DSM-IV y DSM- } \\ \text { IV-TR }\end{array} & \text { DSM-5 CIE-10 }\end{array}$

\begin{tabular}{llllll} 
Denominación & $\begin{array}{l}\text { Trastorno } \\
\text { por déficit de } \\
\text { atención }\end{array}$ & $\begin{array}{l}\text { Trastorno por } \\
\text { déficit de atención } \\
\text { con hiperactividad }\end{array}$ & $\begin{array}{l}\text { Trastorno por } \\
\text { déficit de atención } \\
\text { con hiperactividad }\end{array}$ & $\begin{array}{l}\text { Trastorno por } \\
\text { déficit de atención } \\
\text { con hiperactividad }\end{array}$ & $\begin{array}{l}\text { Trastorno } \\
\text { hipercinético }\end{array}$ \\
\hline Edad de inicio & $\begin{array}{l}\text { Antes de los siete } \\
\text { años (de forma } \\
\text { típica a los tres) }\end{array}$ & $\begin{array}{l}\text { Antes de los siete } \\
\text { años (en la mitad } \\
\text { de los casos antes } \\
\text { de los cuatro } \\
\text { años) }\end{array}$ & $\begin{array}{l}\text { Antes de los siete } \\
\text { años }\end{array}$ & $\begin{array}{l}\text { Algunos síntomas } \\
\text { estaban antes de } \\
\text { los doce años }\end{array}$ & $\begin{array}{l}\text { Antes de los seis } \\
\text { años }\end{array}$ \\
\hline $\begin{array}{l}\text { Duración de } \\
\text { los síntomas }\end{array}$ & $\begin{array}{l}\text { Al menos seis } \\
\text { meses }\end{array}$ & $\begin{array}{l}\text { Al menos seis } \\
\text { meses }\end{array}$ & $\begin{array}{l}\text { Al menos seis } \\
\text { meses }\end{array}$ & $\begin{array}{l}\text { Al menos seis } \\
\text { meses }\end{array}$ & $\begin{array}{l}\text { Al menos seis } \\
\text { meses }\end{array}$ \\
\hline
\end{tabular}


CuADro 2. Comparación de los criterios diagnósticos del TDAH en diferentes versiones del DSM y la CIE-10 (cont.)

\begin{tabular}{|c|c|c|c|c|c|}
\hline & DSM-III & DSM-III-TR & $\begin{array}{l}\text { DSM-IV y DSM- } \\
\text { IV-TR }\end{array}$ & DSM-5 & CIE-10 \\
\hline $\begin{array}{l}\text { Síntomas } \\
\text { centrales }\end{array}$ & $\begin{array}{l}\text { Falta de } \\
\text { atención, } \\
\text { impulsividad e } \\
\text { hiperactividad }\end{array}$ & $\begin{array}{l}\text { Falta de atención, } \\
\text { impulsividad e } \\
\text { hiperactividad }\end{array}$ & $\begin{array}{l}\text { Desatención, e } \\
\text { hiperactividad- } \\
\text { impulsividad }\end{array}$ & $\begin{array}{l}\text { Inatención y/o } \\
\text { hiperactividad- } \\
\text { impulsividad }\end{array}$ & $\begin{array}{l}\text { Falta de } \\
\text { atención e } \\
\text { hiperactividad }\end{array}$ \\
\hline $\begin{array}{l}\mathrm{N}^{0} \text { de síntomas } \\
\text { necesarios } \\
\text { para el } \\
\text { diagnóstico del } \\
\text { trastorno }\end{array}$ & $\begin{array}{l}\text { Al menos tres de } \\
\text { falta de atención } \\
\text { e impulsividad } \\
\text { (de los cinco } \\
\text { propuestos para } \\
\text { desatención } \\
\text { y seis para } \\
\text { impulsividad) } \\
\text { y dos o más de } \\
\text { hiperactividad } \\
\text { (de los cinco } \\
\text { propuestos }\end{array}$ & $\begin{array}{l}\text { Ocho o más } \\
\text { (de los catorce } \\
\text { propuestos) }\end{array}$ & $\begin{array}{l}\text { Seis o más de } \\
\text { desatención o de } \\
\text { hiperactividad- } \\
\text { impulsividad } \\
\text { (de los nueve } \\
\text { propuestos para } \\
\text { cada agrupación) }\end{array}$ & $\begin{array}{l}\text { En pacientes } \\
\text { menores de } 17 \\
\text { años: } 6 \text { síntomas } \\
\text { de inatención } \\
\text { y/o } 6 \text { de } \\
\text { hiperactividad/ } \\
\text { impulsividad. } \\
\text { En pacientes de } \\
17 \text { años o más: } \\
5 \text { síntomas de } \\
\text { inatención y/o } 5 \\
\text { de hiperactividad/ } \\
\text { impulsividad }\end{array}$ & $\begin{array}{l}\text { Requiere la } \\
\text { presencia de la } \\
\text { falta de atención } \\
\text { e hiperactividad } \\
\text { excesivas para } \\
\text { la edad y CI } \\
\text { del afectado. La } \\
\text { desinhibición } \\
\text { es un rasgo } \\
\text { asociado, no } \\
\text { necesario ni } \\
\text { suficiente para } \\
\text { el diagnóstico }\end{array}$ \\
\hline $\begin{array}{l}\text { Necesaria la } \\
\text { presencia de } \\
\text { síntomas en } \\
\text { más de un } \\
\text { contexto }\end{array}$ & No & No & $\begin{array}{l}\text { Sí en dos o más } \\
\text { (casa y escuela, } \\
\text { escuela/ trabajo) }\end{array}$ & $\begin{array}{l}\text { Sí algunos } \\
\text { síntomas en dos } \\
\text { o más (casa y } \\
\text { escuela, escuela/ } \\
\text { trabajo) }\end{array}$ & $\begin{array}{l}\text { Sí en más de } \\
\text { uno (casa, } \\
\text { contexto escolar } \\
\text { o en evaluación } \\
\text { clínica y } \\
\text { psicométrica) }\end{array}$ \\
\hline $\begin{array}{l}\text { Otras áreas } \\
\text { afectadas por } \\
\text { el trastorno }\end{array}$ & $\begin{array}{l}\text { Frecuentemente } \\
\text { el deterioro } \\
\text { escolar y social }\end{array}$ & $\begin{array}{l}\text { Frecuentemente el } \\
\text { deterioro escolar y } \\
\text { social }\end{array}$ & $\begin{array}{l}\text { Actividad social, } \\
\text { académica o } \\
\text { laboral }\end{array}$ & $\begin{array}{l}\text { Actividad social, } \\
\text { académica o } \\
\text { ocupacional }\end{array}$ & $\begin{array}{l}\text { Actividad social, } \\
\text { académica o } \\
\text { laboral }\end{array}$ \\
\hline Subtipos & $\begin{array}{l}\text { - Trastorno } \\
\text { por déficit de } \\
\text { atención con } \\
\text { hiperactividad } \\
\text { - Trastorno } \\
\text { por déficit de } \\
\text { atención sin } \\
\text { hiperactividad } \\
\text { - rastorno por } \\
\text { déficit de } \\
\text { atención tipo } \\
\text { residual }\end{array}$ & $\begin{array}{l}\text { - Trastorno } \\
\text { por déficit } \\
\text { de atención } \\
\text { indiferenciado }\end{array}$ & $\begin{array}{l}\text { - Con predominio } \\
\text { del déficit de } \\
\text { atención } \\
\text { - Con predominio } \\
\text { hiperactivo- } \\
\text { impulsivo } \\
\text { - Tipo combinado } \\
\text { - No especificado }\end{array}$ & $\begin{array}{l}\text { - Con predominio } \\
\text { del déficit de } \\
\text { atención } \\
\text { - Con predominio } \\
\text { hiperactivo- } \\
\text { impulsivo } \\
\text { - Tipo combinado } \\
\text { - TDAH } \\
\text { Especificado } \\
\text { /TDAH No } \\
\text { especificado }\end{array}$ & $\begin{array}{l}\text { - Trastorno de la } \\
\text { actividad y la } \\
\text { atención } \\
\text { - Trastorno } \\
\text { hipercinético } \\
\text { disocial } \\
\text { - Otros } \\
\text { trastornos } \\
\text { hipercinéticos } \\
\text { - Trastorno } \\
\text { hipercinético } \\
\text { sin } \\
\text { especificación }\end{array}$ \\
\hline
\end{tabular}

Fuente: adaptación de Amador, Forns y González, 2010: 55.

El trastorno por déficit de atención — con o sin hiperactividad — ha sido incluido en el DSM-5 como un trastorno del neurodesarrollo, muy frecuente en la población escolar, que va haciéndose visible en todos los ámbitos de desarrollo del niño y que sigue manifestándose a lo largo de su adolescencia e incluso perdura en la edad adulta aunque con distintas manifestaciones.

La triada característica del TDAH la constituye el déficit de atención, la hiperactividad y la impulsividad, y se clasifica en función de los 
síntomas. Estos son utilizados para el diagnóstico, llevando en algunas ocasiones a confusión.

¿Cuál es su etiología? En un principio sus causas eran desconocidas y se fueron atribuyendo a infecciones, traumatismos, daños cerebrales, etc. Estudios realizados por Buchsbaum y Wender en 1973 (citados en González Acosta, 2006) demostraron que la actividad eléctrica del cerebro, medida a través de un encefalograma, en niños con TDAH es menor que en niños que no lo padecen, sobre todo en las áreas frontales. Las distintas investigaciones se han ido sucediendo en el tiempo y aportando datos esclarecedores.

En este sentido, muchos trabajos señalan que los niños con TDAH además de la inatención y del comportamiento hiperactivo-impulsivo manifiestan problemas en las funciones ejecutivas, que afectan la inhibición, memoria de trabajo, planeación, organización, iniciativa, flexibilidad mental, entre otras funciones (p.e., Houghton et al., 1999; Pennington y Ozonoff, 1996; Pineda, Ardila, y Rosselli, 1999; Shallice et al., 2002). Estas dificultades se han puesto en evidencia por medio de pruebas neuropsicológicas específicas. Se sabe que la corteza prefrontal constituye el sustrato esencial del funcionamiento ejecutivo, ya que determina la inhibición activa de las acciones irrelevantes mientras facilita el curso de aquellas dirigidas a un objetivo (Miller y Cohen, 2001). Esto le permite controlar niveles simultáneos del procesamiento, selección, mantenimiento, actualización y reorientación de la conducta, explicando asimismo, la regulación emocional de la misma (Shimamura, 2000).

Esto explicaría la dificultad que tienen los individuos con TDAH para frenar las respuestas impulsivas, para detener las conductas que ya estaban en marcha, para resistir a la interferencia, para organizar de manera secuencial sus actividades y para mantener los esfuerzos cognitivos centrados en una sola actividad (LópezCampo, 2004). Todo ello, se relaciona con el modelo de disfunción ejecutiva en las personas con TDAH (Vaquerizo-Madrid, Estévez-Díaz y Pozo-García, 2005).

Barkley (2002) señala que la lesión en el lóbulo frontal del cerebro, denominada orbito-frontal, es la responsable de los trastornos en la inhibición de la conducta, de problemas para mantener la atención y el autocontrol y de dificultades en la planificación del futuro.

En el 2006 no existía ninguna duda de que el TDAH era un trastorno neurobiológico, en el que influyen factores biológicos y ambientales (Muñoz Yunta, Palau, Salvadó y Valls, 2006: 184). Unos años más y gracias al avance de la sociedad, la ciencia y la medicina, se ha hecho posible que técnicas de neurobiología, fisiopatología, neuroimagen y genética con sus investigaciones determinen que se trata de un trastorno heterogéneo, en el que confluyen además de factores psicobiológicos, otros ambientales y/o educativos. Otros estudios como los realizados con tomografía por emisión de positrones (PET) determinan que las personas que presentan TDAH utilizan menor cantidad de glucosa en las áreas que controlan la atención (Kollins, 2009). Y los realizados por Biederman y Faraone en 1990 (citado en Martínez y Conde, 2009) determinan influencias genéticas en hermanos hasta en un $80 \%$, y sostienen que aproximadamente un $25 \%$ de familiares de primer grado de niños con TDAH, presentan también este trastorno.

Por tanto, los niños con TDAH presentan alteraciones en procesos cognitivos, conductuales y emocionales que dificultan la realización de tareas de la vida cotidiana y la adaptación social (Ramos-Loyo, Taracena, Sánchez-Loyo, Mature y González-Garrido, 2011).

Además de los factores genéticos existen factores ambientales que se han asociado con aumento de riesgo de presentar TDAH (Thapar, Cooper, Jefferies, y Stergiakouli, 2012) y médicos como hipertensión, anemia, sepsis urinaria 
de la madre, partos prolongados, edad gestacional de más de 40 semanas, sufrimiento fetal, que también podrían provocarlo (Guillen, Gracia Marco, Sánchez García y Herreros Rodríguez, 2002).

Durante los últimos 20 años las técnicas de neuroimagen han demostrado que está asociado a alteraciones morfológicas, funcionales y de la conectividad de varios circuitos frontoestriadosparietales y frontocerebrales que intervienen en las funciones ejecutivas (Rubia, Alegría y Brinson, 2014).

La hiperactividad es una de las patologías más estudiadas en los últimos años, y a lo largo de las últimas décadas han proliferado estudios sobre el reparto y la localización con el fin de conocer la prevalencia de este trastorno. Los distintos estudios han demostrado que las tasas son muy variables en todo el mundo que van desde un mínimo de $1 \%$ hasta un máximo de 20\% en niños de edad escolar (Bird, 2002; Faraone, Sergeant, Guillberg y Biederman, 2003), de ahí que sea un tema controvertido pues puede variar dependiendo de los medios diagnósticos, los criterios utilizados e incluso el país donde se realice el estudio (Cardo y Servera,
2005). En España se estima que hay una prevalencia del 5\% (Cardo, Servera y LLobera, 2007), lo que equivaldría a uno o dos niños por aula.

Según la revisión realizada sobre estudios de prevalencia en torno al TDAH, a pesar de tener unos criterios diagnósticos marcados por la CIE o el DSM, todavía podemos observar discrepancias en los porcentajes de prevalencia que nos ofrecen. Las causas de las mismas se pueden valorar en el cuadro 3 donde queda reflejada la disparidad de datos de las últimas décadas y podemos ver diferentes aspectos (criterios, edades...) que contribuyen a estas diferencias. Como podemos observar, en los estudios realizados en diferentes países, la prevalencia va desde el 0,78 al 20,4\% de valores mínimo y máximo recogidos en el cuadro. Esta variabilidad puede deberse tanto al contexto geográfico como al año de realización del estudio, edad de los sujetos de la muestra, así como a los criterios utilizados para realizar el diagnóstico. En este sentido, podemos señalar que el criterio utilizado es determinante en los datos de prevalencia como puede verse claramente si nos fijamos por ejemplo en el caso de Hong Kong o Alemania.

\section{CuAdro 3. Síntesis de estudios sobre prevalencia de TDAH}

\begin{tabular}{|c|c|c|c|c|c|}
\hline Estudio & Año & País & Criterios & Edades & Prevalencia \\
\hline Esser et al. & 1990 & Alemania & ICD-9 & 8 años & $4,2 \%$ \\
\hline Pelhalm et al. & 1992 & EE.UU. & DSM-III-R & Preescolar a $8^{\circ}$ grado & $7,1 \%$ \\
\hline Galluci et al. & 1993 & Italia & DSM-III-R & $4^{\circ}$ grado & $3,9 \%$ \\
\hline Baumgaertel et al. & 1995 & Alemania & $\begin{array}{l}\text { DSM-III-R } \\
\text { DSM-IV }\end{array}$ & $\begin{array}{l}5 \text { a } 12 \text { años } \\
5 \text { a } 12 \text { años }\end{array}$ & $\begin{array}{r}9,6 \% \\
17,8 \%\end{array}$ \\
\hline Leung et al. & 1996 & Hong Kong & $\begin{array}{l}\text { DSM-III } \\
\text { DSM-III-R } \\
\text { CIE-10 }\end{array}$ & $\begin{array}{l}1^{\circ} \text { grado } \\
1^{\circ} \text { grado } \\
1^{\circ} \text { grado }\end{array}$ & $\begin{array}{l}6,1 \% \\
8,9 \% \\
0,78 \%\end{array}$ \\
\hline Verhulst et al. & 1997 & Holanda & DSM-III-R & 13 a 18 años & $7,9 \%$ \\
\hline Gaub y Carlson & 1997 & EE.UU. & DSM-IV & 6 a 9 años & $8 \%$ \\
\hline Gomez et al. & 1999 & Australia & DSM-IV & 5 a 11 años & $2,4 \%$ \\
\hline Guardiola et al. & 2000 & Brasil & DSM-IV & $1^{\circ}$ grado & $18 \%$ \\
\hline
\end{tabular}


CuAdro 3. Síntesis de estudios sobre prevalencia de TDAH (cont.)

\begin{tabular}{lllllc} 
Estudio & Año & País & Criterios & Edades & Prevalencia \\
\hline Rowland et al. & 2001 & EE.UU. & DSM-IV & $1^{\circ}-5^{\circ}$ grado & $16 \%$ \\
\hline Pineda et al. & 2001 & Colombia & DSM-IV & 4 a 7 años & $17,1 \%$ \\
\hline Montiel- Nava et al. & 2002 & Venezuela & DSM-IV & 6 a 12 años & $7 \%$ \\
\hline Cornejo et al. & 2005 & Colombia & DSM-IV & $4-17$ años & $20,4 \%$ \\
\hline Cardo et al. & 2007 & España & ADHD-RS-IV & $6-11$ años & $6,6 \%$ \\
\hline Rodríguez et al. & 2009 & $\begin{array}{l}\text { (Castilla La } \\
\text { Mancha) }\end{array}$ & DSM-IV & $6-16$ años \\
\hline Alloway et al. & 2010 & España & & \\
\hline Jiménez et al. & 2012 & $\begin{array}{l}\text { (Canarias) } \\
\text { España }\end{array}$ & ADHD-RS-IV & $6-12$ años & $4,9 \%$
\end{tabular}

Fuente: extraído y adaptado a partir Díaz, Jiménez, Rodríguez, Alfonso y Artiles (2013: 157).

La comorbilidad de esta patología es muy alta como demuestran numerosos estudios, entre ellos está el realizado por Kadesjo y Gillberg (2001) en el que un $87 \%$ de niños que cumplían criterios del trastorno TDAH tenían al menos un diagnóstico comórbido (Larson y Liverman, 2011). Se puede decir que la comorbilidad en la edad infantil es muy frecuente, pero no hemos de confundir rasgos asociados con trastornos comórbidos.

Los diagnósticos comórbidos reflejados en la bibliografía científica son asociados con mayor frecuencia con trastornos del comportamiento, ansiedad, estado de ánimo, enuresis o tics. Asimismo, los casos de TDAH tienen ciertos rasgos asociados como son: peor rendimiento académico (lectura, matemáticas, expresión escrita), problemas de comportamiento y conducta escolar (relación con los compañeros, competencias de organización, respeto de normas), problemas de desarrollo psicomotor y problemas de relaciones interpersonales. Estos rasgos asociados son factores inherentes a ellos, debido a las disfunciones neuropsicológicas, le dificultan a lo largo de su desarrollo biopsicosocial, y la inmadurez neuroevolutiva se ve reflejada en su desarrollo (Barkley, 2011).

Esta comorbilidad tan alta contribuye a la heterogeneidad en las manifestaciones clínicas y da lugar a un diagnóstico poco específico, en algunas ocasiones.

\section{Consideraciones diagnósticas en TDAH}

En cuanto al diagnóstico del TDAH es una labor compleja, ya que debe realizarse desde un punto de vista multidisciplinar. La forma de evaluar sigue planteando ciertas dudas, hecho que no debe sorprendernos cuando ni en el DSM-IV o el DSM-5 (ver cuadro 4), ni en la CIE-10, aparece una definición al uso de dicha patología y tan solo se enumeran unos rasgos de conducta.

En el DSM-IV TR se nos hace una enumeración de los criterios diagnósticos que se evalúan mediante la observación, dependiendo de la subjetividad del observador y con una valoración subjetiva como "a menudo", "en exceso". 


\section{Cuadro 4. Criterios diagnósticos del trastorno por déficit de atención e hiperactividad}

\begin{tabular}{ll} 
Criterios DSM-IV-TR & DSM-5 \\
\hline
\end{tabular}

\begin{tabular}{|c|c|c|}
\hline A & $\begin{array}{l}\text { La característica principal del TDAH es } \\
\text { un patrón persistente de desatención y /o } \\
\text { hiperactividad/impulsividad, más frecuente } \\
\text { y grave que el observado en sujetos de un nivel } \\
\text { de desarrollo similar }\end{array}$ & El mismo que para DSM-IV-TR \\
\hline B & $\begin{array}{l}\text { Algunos síntomas de hiperactividad-impulsividad } \\
\text { o de desatención causantes de problemas } \\
\text { estuvieron presentes antes de los siete años de } \\
\text { edad; otros sujetos son diagnosticados habiendo } \\
\text { estado presentes los síntomas por varios años }\end{array}$ & $\begin{array}{l}\text { Algunos síntomas de hiperactividad-impulsividad } \\
\text { o de desatención estuvieron presentes antes de } \\
\text { los } 12 \text { años de edad y no es un requisito } \\
\text { el que esos síntomas hayan causado problemas } \\
\text { o discapacidad }\end{array}$ \\
\hline $\mathrm{C}$ & $\begin{array}{l}\text { Algún problema relacionado con los síntomas } \\
\text { debe producirse en dos situaciones por lo menos } \\
\text { (por ejemplo en el hogar y en la escuela o en el } \\
\text { trabajo) }\end{array}$ & El mismo que para DSM-IV-TR \\
\hline $\mathrm{D}$ & $\begin{array}{l}\text { Debe haber pruebas claras o evidencia clínica } \\
\text { de disfunción en la actividad, social, académica } \\
\text { o laboral propia del nivel de desarrollo }\end{array}$ & $\begin{array}{l}\text { No es necesario que se observen pruebas } \\
\text { de disfunción basta con que haya interferencia } \\
\text { o disminución de la calidad de desempeño en } \\
\text { actividades sociales, académicas o laborales }\end{array}$ \\
\hline E & $\begin{array}{l}\text { Los síntomas no se presentan exclusivamente } \\
\text { en el transcurso de un trastorno generalizado } \\
\text { del desarrollo, esquizofrenia u otro trastorno } \\
\text { psicótico, y no se explica mejor por la presencia } \\
\text { de otro trastorno mental como del estado de } \\
\text { ánimo, de ansiedad no disociativo o de la } \\
\text { personalidad }\end{array}$ & $\begin{array}{l}\text { Los síntomas no se presentan exclusivamente en } \\
\text { el trascurso de una esquizofrenia u otro trastorno } \\
\text { psicótico y no se explica mejor por la presencia } \\
\text { de otro trastorno mental }\end{array}$ \\
\hline
\end{tabular}

Fuente: extraído de DSM-IV-TR y DSM-5.

Esto da lugar a dificultades de precisión en el diagnóstico. Entre ambos manuales existen algunas diferencias tales como los subtipos que proponen, la edad de aparición de los síntomas o su asociación con otros trastornos, lo que aún dificulta más el diagnóstico.

Al ser una patología que puede afectar al desarrollo integral del niño y que se mantenga hasta la edad adulta, la Academia Americana de Pediatría publicó en el año 2000 unas recomendaciones clínicas para el diagnóstico y la evaluación del TDAH, con el fin de aunar criterios y metodología a la hora de establecer un diagnóstico (AAP, 2000). En el 2001 publicó otra guía para el tratamiento del niño en la edad escolar (AAP, 2001) y recientemente, tras sucesivas revisiones, publicó la Clinical Practice Guideline for the Diagnosis, Evaluation, and Treatment of Attention-Deficit/ Hyperactivity Disorder in Children and Adolescentsse, en ella han ampliado el rango de edad de las recomendaciones y van desde los 4 a los 18 años (AAP, 2011).

En España también se han editado distintas guías tanto desde el ámbito sanitario como educativo, estas han sido elaboradas por comités de investigación. En ellas se aborda el problema del TDAH desde un punto de vista asequible y van dirigidas a los familiares, educadores y sanitarios que forman parte activa en 
el desarrollo del niño con TDAH. Estas guías sirven de punto de referencia para que la evaluación sea cada vez más homogénea dentro de la heterogeneidad de la propia patología (ver cuadro 5).

El proceso de evaluación normalmente comienza bien por solicitud de los padres (movidos por iniciativa propia o por el colegio) o por el pediatra, al haber notado una conducta inapropiada, sin olvidar que ciertas conductas infantiles son fáciles de confundir con algunos síntomas de TDAH. El proceso es el siguiente:

1. Entrevista: con los padres, profesores, niño.

2. Valoración mediante las escalas de evaluación del comportamiento. Evaluación médico-pediátrica.

3. Valoración por parte del Equipo InfantoJuvenil. Tests y pruebas clínicas.

4. Observación directa del comportamiento.

5. Informe, diagnóstico y tratamiento.
Debido a la falta de coincidencia en la valoración de las pruebas entre padres y profesores (ambos cuestionarios han de dar positivo por exigencias del DSM-IV o de la CIE-10 para tener condición TDAH y poder ser evaluados) es necesario que estas se apoyen en pruebas objetivas de laboratorio y en registros de observación conductual.

Las pruebas clínicas neurológicas permiten descartar posibles comorbilidades. Después debe realizarse un electroencefalograma (EEG) para detectar posibles alteraciones bioeléctricas. Más adelante se realizan pruebas de potenciales cognitivos, test neuropsicológicos y si es posible una magnetoencefalografía (MEG) y una resonancia magnética funcional (RMf).

En los cuadros 6 y 7 se recogen las pruebas comportamentales más usuales (seleccionadas en AACAP, 2007), así como los tests neurológicos más difundidos en la exploración del TDAH (citados por Etchepareborda, Paiva-Barón y Abad, 2009).

\section{CuAdro 5. Síntesis de guías de apoyo para la detección del TDAH en España}

\begin{tabular}{|c|c|c|}
\hline Autor/Fundación & Año & $\begin{array}{l}\text { Características de las guías } \\
\text { Observaciones }\end{array}$ \\
\hline $\begin{array}{l}\text { ADANA: Fundación Ayuda al Déficit } \\
\text { de Atención en Niños, Adolescentes y } \\
\text { Adultos }\end{array}$ & 2006 & $\begin{array}{l}\text { Dirigida a educadores. Finalidad: detección y guía de } \\
\text { intervención. } \\
\text { Base: DSM-IV }\end{array}$ \\
\hline $\begin{array}{l}\text { STILL: Asociación balear de niños con } \\
\text { TDAH }\end{array}$ & 2006 & $\begin{array}{l}\text { Guía práctica con recomendaciones de actuación para los } \\
\text { centros educativos en los casos de alumnos con TDAH }\end{array}$ \\
\hline $\begin{array}{l}\text { Ministerio de Sanidad, Política Social } \\
\text { e Igualdad }\end{array}$ & 2011 & $\begin{array}{l}\text { Guía práctica clínica en el SNS. Aborda el TDAH en } \\
\text { cinco bloques: diagnóstico, instrumentos de evaluación, } \\
\text { tratamiento (psicológico, psicopedagógico, farmacológico, } \\
\text { combinado y tratamiento de la comorbilidad) y aspectos } \\
\text { éticos y legales }\end{array}$ \\
\hline $\begin{array}{l}\text { Ministerio de Educación, Cultura y } \\
\text { Deporte }\end{array}$ & 2012 & $\begin{array}{l}\text { Respuesta educativa para el alumnado con TDAH. } \\
\text { Se describen las características, los materiales, } \\
\text { los objetivos, los contenidos, la metodología y la } \\
\text { temporalización, así como los requisitos para poder llevar } \\
\text { a cabo las propuestas para estos niños }\end{array}$ \\
\hline
\end{tabular}

Fuente: elaboración propia. 
Además de las pruebas comportamentales que se han desarrollado en el extranjero y que se ha ido traduciendo al castellano, ya desde hace unos años, disponemos de una escala que se ha popularizado en la actividad psicopedagógica. Se trata de las Escalas Magallanes de Detección de Déficit de Atención -EMA-D.D.A.- (García-Pérez y Magaz, 2003, 2006). El EMAD.D.A. es un instrumento de observación conductual que permite identificar niños que puedan poseer condiciones de base biológica conocidas como TDA o TDAH. Esta escala es la que hemos utilizado en el presente trabajo y está compuesta por dos pruebas el EMA-D.D.A (padres) y el EMA-D.D.A. (profesores). Con ellas se puede evaluar: el déficit de atención sostenida a tareas motrices y cognitivas (reflexividad) e hiperkinesia-hiperactividad (DAH), el déficit de eficacia atencional y lentitud motriz y cognitiva (DA), la agresividad, el retraimiento social, la ansiedad, y el rendimiento académico.

Como resultado del análisis de los datos obtenidos a nivel cuantitativo, nos permite conocer la presencia de condición TDA o TDAH y el análisis cualitativo de esos mismos datos nos permite averiguar, dentro de cada dimensión, los problemas de los alumnos que presentan puntuaciones elevadas en cada una de ellas. Son, pues, escalas muy útiles y de fácil manejo en el ámbito escolar:

En este sentido, y considerando la importancia que tiene el diagnóstico precoz y la dificultad para llevar a cabo pruebas neurobiológicas, pruebas genéticas o análisis bioquímicos que puedan determinar si un niño presenta o no

\section{CuAdro 6. Resumen de las escalas de valoración del comportamiento más utilizadas en la evaluación y el tratamiento del TDAH}

\begin{tabular}{lll} 
Autor & Año & Instrumento \\
\hline Barkley, & & \\
DuPaul et al., & 1990 & Academic Performance Rating Scale (APRS) \\
Brown & 1998 & ADHD Rating Scale-IV \\
Achenbach y Edelbrock & 1991 & Child Behavior Checklist (CBCL) \\
Conners & 1997 & Conners Parent Rating Scale y Revised (CPRSR). \\
Conners y Wells & 1997 & Conners Wells Adolescent Self-Report Scale \\
Conners & 1997 & Conners Teacher Rating Scale y Revised (CTRS-R) \\
Barkley & 1990 & Home Situations Questionnaire y Revised (HSQ-R) \\
Barkley & 1990 & School Situations Questionnaire y Revised (SSQ-R) \\
Loney y Milich & 1982 & Inattention/Overactivity With Aggression (IOWA) \\
Swanson & 1992 & SNAP-IV \\
Wolraich et al. & $2003^{\text {a }}$ & Vanderbilt ADHD Diagnostic Parent and Teacher Scales \\
Ullmann y cols. & $1985^{a}$ & ADD-H: Comprehensive Teacher Rating Scale (ACTeRS) \\
Barkley, 1990 & 1990 & Child Attention Problems (CAP) \\
Wolraich y cols. & 1996 & AD/HD Diagnostic Teacher Rating Scale
\end{tabular}

Fuente: American Academy of Child and Adolescent Psychiatry (AACAP), 2007: 899 
TDAH, debemos basarnos en los estudios de comportamiento para valorar la intensidad de la conducta del niño y concluir si esta es propia de su edad o no. Estos estudios de comportamiento han de realizarse en distintos ambientes y los más propicios por su interacción con el niño son: la familia, la escuela y el grupo de iguales, dado que es donde el niño pasa más horas y con quienes se desenvuelve con mayor naturalidad. De ahí el interés de centrar este estudio en el funcionamiento diferencial de la identificación que realizan padres y profesorado.

\section{Estudio empírico}

\section{Planteamiento del problema y objetivos}

La detección precoz de un TDAH es fundamental para poder conseguir mejores niveles de éxito en la intervención, y evitar que esta problemática se vaya, en su desarrollo, asociando a otros factores. La complejidad del diagnóstico aconseja que la detección escolar sea muy sólida. Y, en este sentido, habitualmente se fundamenta en la identificación que se lleva a cabo a partir de las valoraciones que realizan del comportamiento del niño sus padres y el profesorado. La escala EMA-D.D.A (García-Pérez y Magaz, 2006) se ha difundido en el ámbito escolar como un buen instrumento de ayuda a la detección. Incluye escalas para padres y profesorado, y presentan buenas propiedades métricas.

La Escala EMA-DDA está compuesta por seis dimensiones (hiperactividad, inatención, agresividad, retraimiento, ansiedad y rendimiento) compuestas por ítems que se responden con una escala de 4 puntos (casi nunca, pocas veces, a menudo y casi siempre). Para la corrección se utiliza la escala Likert 0-3 teniendo en cuenta la presencia o ausencia del rasgo que se está evaluando. Así pues, $\mathrm{CN}=0, \mathrm{PV}=1 ; \mathrm{AM}=2$ y $\mathrm{CS}=3$. El valor sobre las puntuaciones directas será 0 en el caso que se den valores $0-1$ y 1 en

\section{CuADro 7. Tests neuropsicológicos más usuales en la exploración del TDAH}

Test

Utilidad

Continuous Performance Test (CPT)

Reaction Time (RT)

Selective Attention Test (SAT)

Test de Stroop

Test de selecciones de cartas de Wisconsin (WCST)

Factor de 'distractibilidad' del WISC-R

Test de emparejamiento de figuras familiares (MFF) o test de Kagan

Figura compleja de Rey
Permite evaluar la atención sostenida y la capacidad de control motor

Evalúa la capacidad de reacción o estado de alerta auditivo y visual Evalúa la atención selectiva o la distinción de la atención

Evalúa el control inhibitorio de interferencia frente a estímulos con doble input

Evalúa la flexibilidad cognitiva

Evalúa aritmética, dígitos y claves, en especial la prueba de claves; es una buena medida para evaluar la atención selectiva

Mide el estilo cognitivo y la capacidad de reflexión-impulsividad

Investigar la organización perceptual y la memoria visual en individuos con lesión cerebral

Fuente: Etchepareborda, Paiva-Barón y Abad (2009). 
el caso de valores 3-4. La puntuación total de la dimensión es la suma de las puntuaciones corregidas.

Los ítems están distribuidos de la siguiente forma:

- Dimensión1: déficit de atención con hiperactividad (DAH), compuesta por diez ítems.

- Dimensión 2: el déficit de eficacia atencional y lentitud motriz y cognitiva (DA), formada por siete ítems.

- Dimensión 3: agresividad la integran, constituida por doce ítems.

- Dimensión 4: se mide el retraimiento con diez ítems.

- Dimensión 5: está conformada por seis ítems.

- Dimensión 6: rendimiento académico medido por doce ítems.

No obstante, existen estudios (Amador, Forns y Martorell, 2001; Trujillo y Díaz-Sibaja, 2007) que señalan que las nominaciones que realizan padres y profesorado presentan diferencias. Para la identificación de condición de TDAH se requiere que esta se pueda señalar a partir de la valoración que realizan tanto padres como profesorado (DSM-IV/V).

De este modo, el objetivo de este estudio es analizar si se dan patrones diferenciales en la valoración que realizan padres y profesorado a partir de las escalas EMA-D.D.A en niños de entre 6 y 9 años de edad (escolarizados en Cuenca — ciudad y provincia-), por lo que se analiza si existe concurrencia entre las valoraciones que emiten ambos colectivos.

\section{Metodología}

Población y muestra: participantes

El estudio forma parte de una investigación más amplia que fue dirigida a la totalidad del alumnado de entre 6 y 9 años de edad $\left(1^{\circ}\right.$ a $4^{\circ}$ de educación primaria) de la provincia de Cuenca, la población de referencia son los niños escolarizados en esta provincia, con la finalidad de identificar el alumnado con posible TDA-H. En la tabla 1 se sintetizan las respuestas obtenidas desde los centros educativos. En cuanto al alumnado de la población de referencia sobre la que finalmente trabajamos es de 2.385 alumnos, que han sido valorados con la Escala EMA-D.D.A. bien sea por el profesorado, por los padres o por ambos.

Atendiendo al objetivo de estudio del que informamos en este trabajo, hay que señalar que un problema habitual en este tipo de investigaciones, cuando se trabaja con información recogida a partir de situaciones reales (expedientes de centros educativos o gabinetes psicopedagógicos) es precisamente que los expedientes diagnósticos no están completos. De este modo, en este caso, la muestra real sobre la que realizamos este estudio es de 331 valoraciones completas en todas las dimensiones de la Escala ${ }^{1}$, es decir, que incluyan la de profesorado y la de padres. Esta mortalidad experimental, afecta en cuanto a la representatividad total que no supera el 95\%, y el error alcanza el $5,16 \%$, con un nivel de heterogeneidad de 50 . Otras posibles fuentes de sesgo se deben al hecho de que no se ha podido controlar la representatividad proporcional en diferentes estratos de generalidad (localidad, sexo, edad, curso...). Sin duda, ello constituye una limitación, pero que representa el modo en que se trabaja en los centros, en los que los profesionales deben atender un elevado número de casos, superior al tiempo de que disponen, lo que se traduce en prácticas deficientes, sobre todo al utilizar los instrumentos diagnósticos que no son aplicados de manera rigurosa según sus instrucciones de diseño. Por ello, somos conscientes de que se trata de un grupo de estudio que, aunque por número total de casos pudiera considerarse estadísticamente representativo, estimamos que el sesgo que se introduce por el modo de reclutamiento de sujetos 
es lo suficientemente importante como para señalarlo explícitamente. En la misma tabla 1 se aportan los datos de la muestra de estudio, cuyo $\mathrm{N}$ totales de 331 casos valorados por ambos colectivos (padres y docentes) y se recoge el número de casos (alumnado) que ha constituido la muestra de referencia, que ha sido facilitada por los centros escolares que se indican.

En cuanto a los análisis de datos realizados para llevar a cabo el análisis diferencial entre las valoraciones emitidas por padres y profesores se ha enfocado desde dos perspectivas: correlacional, mediante el coeficiente de correlación de Pearson $(\mathrm{Rxy})^{2}$, y contraste de medias mediante las pruebas $t$ de Student y U de Mann-Whitney. Para ello se ha utilizado el paquete estadístico SPSS, v.19, bajo licencia de la Universitat de Valéncia.

En el primer caso, se intenta analizar si los patrones de valoración emitidos por padres y profesores siguen un perfil similar, mientras que en el segundo caso se pretende estudiar sin los niveles que se aprecian en cada una de las dimensiones por parte de ambos colectivos, son similares o no. Utilizamos dos procedimientos

TABla 1. Población de referencia y composición de la muestra (alumnado y centros) en el estudio global

\begin{tabular}{lcc} 
& Alumnado & Colegios \\
\hline Población total (referencia) & 4.039 & 72 \\
\hline Provincia & 1.534 & 31 \\
\hline Cuenca & 851 & 11 \\
\hline Negativos (no participan) & 625 & 16 \\
\hline Total de participantes (muestra estudio completo) & 2.385 & 42 \\
\end{tabular}

Muestra específica con la que se trabaja el objetivo que se analiza en este estudio: alumnado valorado por ambos colectivos (padres y docentes)

\begin{tabular}{|c|c|c|c|c|c|}
\hline \multirow[t]{6}{*}{ Edad } & Total & Chicos & Chicas & \multicolumn{2}{|c|}{$\mathrm{Ns} / \mathrm{Nc}$} \\
\hline & 30,5 & $31,9 \%$ & $34,5 \%$ & \multicolumn{2}{|c|}{$7,20 \%$} \\
\hline & 43,7 & $49,4 \%$ & $41,8 \%$ & & \\
\hline & 16,8 & $17,5 \%$ & $20,9 \%$ & & \\
\hline & 1,5 &, $6 \%$ & $2,7 \%$ & & \\
\hline &, 3 &, $6 \%$ & -- & & \\
\hline \multirow[t]{4}{*}{ Curso } & & $47,2 \%$ & $48,5 \%$ & \multicolumn{2}{|c|}{$10,20 \%$} \\
\hline & & $50,9 \%$ & $49,5 \%$ & & \\
\hline & & $1,2 \%$ & $1,9 \%$ & & \\
\hline & & ,6\% & -- & & \\
\hline \multirow[t]{2}{*}{ Sexo } & & & Chicos & Chicas & $\mathrm{Ns} / \mathrm{Nc}$ \\
\hline & & & $53,00 \%$ & $36,20 \%$ & $10,80 \%$ \\
\hline
\end{tabular}


de contraste de hipótesis, dado que en algunas de las dimensiones, se incumple algún supuesto de aplicabilidad de pruebas paramétricas. Por lo que la doble vertiente analítica la asumimos como un medio de validación de resultados.

Asimismo, y como garantía acerca de la fiabilidad de las escalas, se ha realizado un análisis basado en Teoría de Respuesta al Ítem —Modelo de Rasch- a partir de los datos recogidos. En este trabajo informamos acerca de la consistencia interna para cada una de las subescalas (fiabilidad entre ítems) que componen las escalas de padres y profesorado, mediante el coeficiente Alfa de Cronbach (en su especificación como KuderRichardson-20/KR-20). Para ello se ha utilizado el paquete estadístico Winsteps (Linacre, 1991, 2006a y b; González-Montesinos, 2008).

\section{Resultados}

Antes de informar acerca del funcionamiento diferencial de las Escalas entre padres y profesorado, pasamos a comentar brevemente los coeficientes de consistencia interna obtenidos con la muestra de estudio. Los niveles de fiabilidad estimados mediante KR-20 (tabla 2) son ampliamente satisfactorios, por lo que puede asumirse como una garantía para el análisis posterior realizado.

En relación al análisis diferencial (tabla 3), de correlaciones entre totales de dimensiones, en general las correlaciones entre el patrón de valoración de padres y profesores, son bajas o incluso en algunos casos negativas, aunque bajas también y estadísticamente significativas.

En términos generales, sin embargo, como puede observarse en la tabla 3 las correlaciones obtenidas son bajas o medio bajas, si bien significativas, a excepción de la correspondiente a la dimensión de ansiedad. La única dimensión en la que se produce una correlación de mayor intensidad es en la de rendimiento. Obviamente, se trata de un aspecto más objetivable y que se valora en sí mismo, con independencia del contexto en el que se produzca el comportamiento.

Por otra parte, en cuanto a las pruebas $t$ de Student (ver tabla 4) entre las medias de las valoraciones que en cada dimensión realizan padres y profesores, se puede observar que en todas las dimensiones, salvo en la de rendimiento, se observan diferencias estadísticamente significativas.

TABLA 2. Valores de KR-20 para todas las subescalas de la EMA-D.D.A, para padres y profesorado, obtenidos con la muestra de estudio

\begin{tabular}{lcc} 
& \multicolumn{2}{c}{ Colectivos de respondientes } \\
\cline { 2 - 3 } Dimensiones & Padres & Profesorado \\
\hline Hiperactividad & 0,98 & 0,95 \\
\hline Inatención & 0,97 & 0,93 \\
\hline Agresividad & 0,99 & 0,96 \\
\hline Retraimiento & 0,95 & 0,94 \\
\hline Ansiedad & 0,90 & 0,93 \\
\hline Rendimiento & 0,97 & 0,92 \\
\hline
\end{tabular}


TABLA 3. Correlaciones entre las valoraciones de las dimensiones emitidas por padres y profesores

Pad./Prof. Pad./Prof. Pad./Prof. Pad./Prof. Pad./Prof. Prof hiperactividad inatención agresividad retraimiento ansiedad rendimiento

\begin{tabular}{|c|c|c|c|c|c|c|}
\hline Rxy Pearson & ,209 & -120 & , 140 &, 244 & ,090 &, 548 \\
\hline Sig. &, 000 & ,029 & ,010 & ,000 & , 102 & ,000 \\
\hline $\mathrm{N}$ & 334 & 333 & 333 & 333 & 331 & 331 \\
\hline
\end{tabular}

TABLa 4. Síntesis de análisis diferencial mediante prueba t de Student entre valoraciones emitidas por padres y profesores en las diferentes dimensiones

\begin{tabular}{|c|c|c|c|c|c|c|}
\hline Observador & Media & $\begin{array}{l}\text { Desviación } \\
\text { típ. }\end{array}$ & $\begin{array}{l}\text { Error típico } \\
\text { de la media }\end{array}$ & $\begin{array}{l}\text { ¿Hay } \\
\text { diferencia } \\
\text { de varianzas } \\
\text { entre } \\
\text { padres y } \\
\text { profesorado? } \\
\text { (Prueba de } \\
\text { Levène) }\end{array}$ & $\begin{array}{l}\text { ¿Hay } \\
\text { diferencia } \\
\text { entre } \\
\text { padres y } \\
\text { profesorado? } \\
\text { (t de Student) }\end{array}$ & $\begin{array}{l}\text { ¿Hay } \\
\text { diferencia } \\
\text { de entre } \\
\text { padres y } \\
\text { profesorado? } \\
\text { (Prueba U) }\end{array}$ \\
\hline
\end{tabular}

\begin{tabular}{|c|c|c|c|c|c|c|c|}
\hline \multirow[t]{2}{*}{ Hiperactividad } & Padres & 6,82 & 2,470 & , 135 & \multirow[t]{2}{*}{$\mathrm{Si}^{* * *}$} & \multirow[t]{2}{*}{ Sí *** } & \multirow[t]{2}{*}{ Sí *** } \\
\hline & Profesores & 3,61 & 3,471 & , 190 & & & \\
\hline \multirow[t]{2}{*}{ Inatención } & Padres & 1,96 & 2,038 & ,112 & \multirow[t]{2}{*}{ Sí *** } & \multirow[t]{2}{*}{ Sí *** } & \multirow[t]{2}{*}{ Sí *** } \\
\hline & Profesores & 2,53 & 2,397 & ,131 & & & \\
\hline \multirow[t]{2}{*}{ Agresividad } & Padres & 4,10 & 2,529 & , 139 & \multirow[t]{2}{*}{ No } & \multirow[t]{2}{*}{ Sí *** } & \multirow[t]{2}{*}{ Sí *** } \\
\hline & Profesores & 1,51 & 2,556 & , 140 & & & \\
\hline \multirow[t]{2}{*}{ Retraimiento } & Padres & 1,23 & 1,564 & ,086 & \multirow[t]{2}{*}{ No } & \multirow[t]{2}{*}{ Sí *** } & \multirow[t]{2}{*}{ Sí *** } \\
\hline & Profesores & ,83 & 1,446 & ,079 & & & \\
\hline \multirow[t]{2}{*}{ Ansiedad } & Padres &, 72 & ,892 & ,049 & \multirow[t]{2}{*}{ No } & \multirow[t]{2}{*}{ Sí $* * *$} & \multirow[t]{2}{*}{ Sí $* * *$} \\
\hline & Profesores &, 45 & ,964 & ,053 & & & \\
\hline \multirow[t]{2}{*}{ Rendimiento } & Padres & 4,21 & 3,292 & ,181 & \multirow[t]{2}{*}{ No } & \multirow[t]{2}{*}{ No } & \multirow[t]{2}{*}{ No } \\
\hline & Profesores & 4,08 & 3,874 & ,212 & & & \\
\hline
\end{tabular}

$* * * p \leq 0,000$

Normalmente son los padres los que ofrecen valoraciones más elevadas en la mayor parte de las dimensiones, salvo en la de inatención (ver gráfico 3) donde la media de valoración que ofrecen los padres es de 1,96, mientras que la de los profesores es de 2,53.
En las restantes dimensiones, en la que se da más distancia bruta entre la media de valoraciones de profesores y padres es en la de hiperactividad. En este caso, el promedio que ofrecen los padres es de 6,82 , mientras el que dan los profesores es de 3,61. 
GrÁFICo 3. Valoraciones medias de padres y profesorado en todas las subescalas

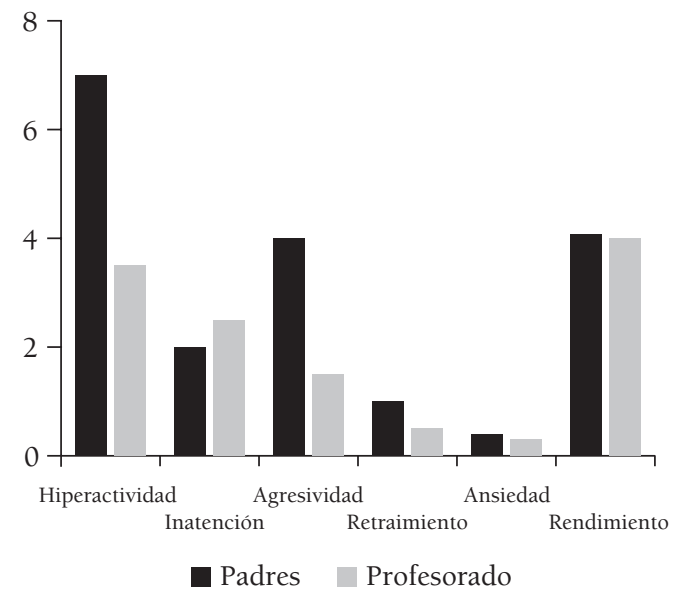

A continuación, destacar que también se observa una distancia considerable entre las valoraciones medias de los dos grupos en la dimensión de agresividad, en la que el promedio de valoraciones de los padres es de 4,10 y de profesores es de 1,51.

En las que menos distancia se observa es en la de ansiedad (Media de profesorado 0,72; padres: $0,45)$; y en la de retraimiento, en la que la media de valoraciones del profesorado es de 0,23 y la de los padres es de 0,83.

En el caso de la dimensión de Rendimiento, la diferencia entre las medias que ofrecen ambos grupos no es estadísticamente significativa, tal como hemos señalado anteriormente. La media del profesorado es 4,21 y la de padres es de 4,08 .

Finalmente, destacar, que realizada la prueba de Levène de contraste de varianzas, se puede observar que normalmente no se dan diferencias significativas entre la variación de ambos grupos. Tan solo en el caso de las dimensiones de hiperactividad e inatención presentan diferencias estadísticamente significativas. En ambos casos es el grupo de profesorado el que presenta mayor heterogeneidad en sus valoraciones.

Respecto al contraste analizado mediante la prueba U de Mann-Whitney se observan los mismos resultados que los obtenidos con la prueba t. En este sentido, se confirman las tendencias comentadas (ver tablas 3, 4 y 5).

\section{Conclusiones}

Según arrojan los datos analizados en nuestro trabajo, no existe mucha concordancia entre las fuentes de información padres-profesores y se aprecian notables diferencias entre la información proporcionada en relación al comportamiento de un niño con posible TDAH, así lo recogen también distintas investigaciones (Díaz-Sibaja y Trujillo, 2008; Acle y Ortiz, 2006).

TABLA 5. Resultados del análisis de contraste no-paramétrico (prueba de U de Mann-Whitney)

Hiperactividad Inatención Agresividad Retraimiento Ansiedad Rendimiento

\begin{tabular}{lrrrrrr} 
U de Mann-Whitney & 27536,000 & 49119,000 & 20600,500 & 44178,000 & 42602,000 & 52426,500 \\
\hline W de Wilcoxon & 83481,000 & 104730,000 & 76545,500 & 100123,000 & 98213,000 & 108037,500 \\
\hline Z & $-11,387$ & $-2,666$ & $-14,312$ & $-5,002$ & $-5,963$ & $-1,162$ \\
\hline Sig. asintót. (bilateral) &, 000 &, 008 &, 000 &, 000 &, 000 &, 245
\end{tabular}


Las diferencias en la información aportada por padres y profesores no necesariamente implican que una sea más válida que la otra. Podemos interpretarlo como un reflejo de las diferencias conductuales ocasionadas por los diversos contextos o a las interacciones diferenciales entre el niño y quienes informan, así como el grado de expectativas o el nivel de tolerancia (Steele, Forehand, y Devine, 1996, Orjales, 2001).

En la valoración de los datos obtenidos hemos de tener en cuenta que los evaluadores responden en función de los contextos y las situaciones en las que se encuentran inmersos. Por ello es importante que se tengan en cuenta las exigencias y características del contexto donde se desarrolla el niño sobre el que se informa (Amador y Forns, 2001).

Según los criterios del DSM-IV, una de las premisas que deben cumplirse para diagnosticar TDAH es que los síntomas deben darse en dos o más ambientes (APA, 1995). La primera parte del diagnóstico de TDAH se va a hacer a través de escalas de observación conductual, por lo que se hace imprescindible la colaboración de padres y profesores en este proceso, ya que son los que mayor tiempo comparten con los niños. Para obtener descripciones válidas sobre la conducta del niño, los padres y profesores son las mejores fuentes de información, para la mayoría de los trastornos externos (MontielNava y Peña, 2001).

Herjanic y Reich (1982), al igual que Verhulst y Van der Ende (1991), afirman que los profesores y los padres son mejores fuentes de información y con una concordancia entre observadores del mismo grupo (padres o profesores) más elevada, cuando el área que debe evaluarse incluye conductas externas; mientras que esta consistencia disminuye cuando se evalúan problemas internos.

Considerando los resultados obtenidos, la dimensión que obtiene un mejor nivel de correlación es la de rendimiento, lo que quiere decir que tanto padres como profesores coinciden a la hora de valorar el rendimiento académico de estos alumnos. Algo que es de esperar ya que estos chicos presentan un rendimiento académico insuficiente e insatisfactorio, debido a que no logran los conocimientos escolares requeridos para el grado que cursan (Portellano, 1989; Corral, 2005), por lo que necesitan del seguimiento y la ayuda tanto de padres como de profesores, hecho que explica esta coincidencia.

Por otra parte, se aprecian notables diferencias entre la información proporcionada por los padres y por los profesores en relación al comportamiento de un niño con posible TDAH (Díaz-Sibaja y Trujillo, 2008; Acle y Ortiz, 2006). Así, observamos que los padres ofrecen valoraciones más elevadas que los profesores. Se aporta evidencia de la tendencia a puntuar más alto de los padres, asumiendo que estos tienen un menor número de referencias para comparar a sus hijos que las que pudieran tener los profesores.

En el caso de la inatención la media de valoración del profesorado es bastante más alta que la de los padres, algo que es de esperar, puesto que en casa la demanda de atención sostenida es menos frecuente que en clase, donde la mayoría de actividades requieren este tipo de atención.

En hiperactividad y agresividad, ocurre lo contrario que con los problemas de atención, destacan las puntuaciones de los padres sobre las de los profesores. Estamos de acuerdo con Amador, en que a medida que se pasa de preescolar a ciclos superiores se incrementa la frecuencia de las conductas relacionadas con hiperactividad. En este sentido, en los primeros cursos hasta que aprenden a acatar normas es frecuente que el profesorado considere excesivo el nivel de movimiento detectado en los alumnos. Este exceso de movimiento se controlará notablemente en aquellos casos que el principal problema sea simplemente 
instruccional, no así en posibles casos de condición TDAH. Las valoraciones realizadas por padres y profesores nos sugieren que al subir de nivel se les exige a los alumnos un mayor esfuerzo para controlar su actividad excesiva, y que padres y profesores juzgan con mayor rigidez esta falta de control (Amador et al., 2006). El alumnado no se comporta igual en el entorno familiar donde no hay reglas en cuanto al exceso de movimiento que en el colegio donde hay unas normas que respetar, las cuales se han exigido desde su inicio en el centro. Al igual ocurre con la agresividad: las normas de comportamiento preestablecidas condicionan su actitud en el centro escolar.

\section{Notas}

${ }^{1}$ En algunas dimensiones es ligeramente mayor, como, por ejemplo, en la de hiperactividad de 334 casos.

${ }^{2}$ Téngase en cuenta que, si bien las valoraciones que ofrecen padres y profesorado en cada ítem están en una escala Likert de 4 puntos (en la que puede asegurarse únicamente la ordinalidad), la Escala no trabaja con estos puntajes directos. Se corrige, como si se trata de una prueba de logro, en la que en función de la respuesta al ítem, se consigna presencia/ausencia en la dimensión y el puntaje total de cada dimensión es la suma que se adecua a una escala 0-10. En este sentido, asumimos que se trata de una métrica asimilable a la de intervalo, por lo que trabajamos con $r_{x y \text { de Pearson. }}$.

\section{Referencias bibliográficas}

Acle, G., y Ortiz, J. A. (2006). Diferencias entre padres y maestros en la identificación de síntomas del trastorno por déficit de atención con hiperactividad en niños mexicanos. Revista de Neurología, 42 (1), 17-21.

Amador, J. A., y Forns, M. (2001). Sensibilidad y especificidad de la valoración de padres y profesores de los síntomas del Trastorno por déficit de atención con hiperactividad. Anuario de Psicología, 32 (4), 65-78.

Amador, J. A., Forns, M., y González, M. (2010). Trastorno por déficit de atención con hiperactividad (TDAH). Madrid: Síntesis S.A.

Amador, J. A., Forns, M., y Martorell, B. (2001). Síntomas de desatención e hiperactividad-impulsividad: análisis evolutivo y consistencia entre informantes. Anuario de Psicología, 32 (1), 51-66.

Amador, J. A., Forns, M., Guàrdia, J., y Peró, M. (2006). Estructura factorial y datos descriptivos del perfil de atención y del cuestionario TDAH para niños en edad escolar. Psicothema, 18 (4), 696703.

American Academy of Child and Adolescent Psychiatry (AACAP) (2007). Practice parameter for the assessment and treatment of children and adolescents with attention-deficit / hyperactivity disorder. Journal of American. Academy of Child \& Adolescent Psychiatry, 46 (7), 894-921.

American Academy of Pediatrics (AAP) (2000). Clinical Practice Guideline: Diagnosis and evaluation of the child with attention-deficit/hyperactivity disorder. Pediatrics, 105 (5), 1158-1170.

American Academy of Pediatrics (AAP) (2001). Clinical practice guideline: Treatment of the School-Aged Child with Attention-deficit/hyperactivity disorder. Pediatrics, 108 (4), 10331044.

American Academy of Pediatrics (2011). ADHD: Clinical practice guideline for the diagnosis, evaluation, and treatment of attention-deficit/hyperactivity disorder in Children and Adolescents. Pediatrics, 128 (5), 1007-1024. 
American Psychiatric Association (2002). DSM-IV-TR. Manual diagnóstico y estadístico de los trastornos mentales. Barcelona: Masson.

American Psychiatric Association (2013). DSM- 5. Diagnostic and statistical manual of mental disorders. Washington: American Psychiatric Association.

Barkley, R. A. (2002). International consensus statement on ADHD. Clin child Fam Psychology Review, 5 (2), 89-111

Barkley, R. A. (2011). Niños hiperactivos: cómo comprender y atender sus necesidades especiales. Guía completa del trastorno por déficit de atención con hiperactividad (TDAH). Barcelona: Paidós.

Biederman, J., Rosenbaum, J. F., Hirshfeld, D. R., Faraone, S. V., Bolduc, E. A., Gersten, M., Meminger, S. R., Kagan, J., Snidman, N., Reznick, J. S., y Reznick, PhD. (1990). Psychiatric correlates of behavioral inhibition in young children of parents with and without psychiatric disorders. Arch Gen Psychiatry, 47 (1), 21-26.

Bird, H. R. (2002). The diagnostic classification, epidemiology and cross-cultural validity of ADHD, in Attention Deficit Hyperactivity Disorder: State of the Science: Best Practices. Kingston, NJ: Jensen PCJ.

Buchsbaum, M., y Wender, P. (1973). Average evoked responses in normal and minimally brain dysfunctioned children treated with amphetamine. Arch Gen Psychiatry, 29 (6), 764-770.

Cardo, E., y Servera, M. (2005). Prevalencia del trastorno por déficit de atención e hiperactividad. Revista Neurología, 40 (Supl. 1), S11-5.

Cardo, E., Servera, M., y Llobera, J. (2007). Estimación de la prevalencia de déficit de atención con hiperactividad entre la población de la isla de Mallorca. Revista de Neurología, 44, 10-14.

Centers for Disease Control and Prevention CDC (2014). Data and Statistics in the United States. Recuperado de: http://www.cdc.gov

Corral, P. (2005). La hiperactividad infantil y juvenil. En M. Comeche y M. Vallejo (eds.), Manual de Terapia de la conducta en la infancia (pp. 519-549). Madrid: DyKinson.

Díaz, A., Jiménez, J. E., Rodríguez, C., Alfonso, M., y Artiles, C. (2013). Consideraciones de los estudios de prevalencia del trastorno por déficit de atención con o sin hiperactividad (TDAH). Revista de Psicología y Educación, 8 (2), 155-170.

Díaz-Sibaja M. A., y Trujillo, A. (2008). Eficacia de un protocolo de evaluación multi-metodológico para el diagnóstico del trastorno por déficit de atención con hiperactividad. Revista de Psiquiatría y psicología del niño y del adolescente, 8 (1), 1-11.

Etchepareborda, M. C., Paiva-Barón, H., y Abad, L. (2009). Ventajas de las baterías de exploración neuropsicológicas en el trastorno por déficit de atención/hiperactividad. Revista de Neurología, $48, n^{\circ}$ extra 2, 89-93.

Faraone, S. V., Sergeant, J., Gillberg, C., y Biederman, J. (2003). The worldwide prevalence of ADHD: is it an American condition? World Psychiatry, 2, 104-113.

García-Pérez, M., y Magaz, A. (2003). Hiperactividad. Guía para profesores. Cruces-Baracaldo: ALBOR- COHS.

García-Pérez, M., y Magaz, A. (2006). Escala Magallanes de Detección de Déficit de Atención: EMADDA Burceña-Barakaldo: ALBOR-COHS.

González Acosta, E. (2006). Trastorno de déficit de atención e hiperactividad en el salón de clases. Tesis doctoral. Universidad Complutense de Madrid, Madrid.

González Montesinos, M. J. (2008). Análisis de Reactivos a través del Modelo de Rasch. Manual Técnico A. Serie Medición y Metodología. México, DF: INEE -UNISON.

Guillén Pino, F., Gracia Marco, R., Sánchez García, F., y Herreros Rodríguez, O. (2002). Trastorno de déficit de atención con hiperactividad (TDAH): puesta al día. Centro de salud, 10 (9), 536542. 
Herjanic, B., y Reich, W. (1982). Development of a structured psychiatric interview for children: agreement between child and parent on individual symptoms. J. Abnorm Child Psychol, 10 (3), 307-324.

Houghton, S., Douglas, G., West, J., Whiting, K., Wall, M., Langsgord, S., Powell, L., y Carroll, A. (1999). Differential patterns of executive function in children with Attention-Deficit Hyperactivity Disorder according to gender and subtype. Journal of Child Neurology, 14, 801-805.

Kadesjö, B., y Gillberq, C. (2001). The comorbidity of ADHD in the general population of Swedish school-age children. J Child Psychol Psychiatry, May 42 (4), 487-492.

Kollins, S. (2009). Genética, neurobiología y neurofarmacología del trastorno por déficit de atención e hiperactividad (TDAH). RET, Revista de toxicomanías, 55, 19-27.

Larson, E. L., y Liverman, C. T. (2011). Preventing transmission of pandemic influenza and other viral respiratory diseases: personal protective equipment for healthcare workers: Update 2010. Washington, DC: National Academies Press.

Linacre, J. M. (1991-2006a). Rasch-Model Computer Programs @), Chicago, IL: Electronic Publication. http://www.winsteps.com

Linacre, J. M. (2006b). A User's Guide to Winsteps Ministeps: Rasch-Model Computer Programs. Chicago, IL: Electronic Publication. www.winsteps.com

Linacre, J. M. (2013). Facets computer program for many-facet Rasch measurement, version 3, 71.2. Beaverton, Oregon: Winsteps.com.

López-Campos, G. X., Gómez-Betancur, L. A., Aguirre-Acevedo, D. C., Puerta, I. C., y Pineda, D. C. (2005). Componentes de las pruebas de atención y función ejecutiva en niños con trastorno por déficit de atención/hiperactividad. Revista de Neurología, 40, 331-339.

Martínez, M. A., y Conde, H. (2009). Trabajar con personas con TDAH, una labor de equipo. Tesis Doctoral. Burgos: Universidad de Burgos.

Martínez-Frutos, M. T., Herrera-Gutiérrez, E., y Peyres-Egea, C. (2014). Los conocimientos del profesorado sobre TDAH y su relación con el rendimiento del alumnado. En J. Maquilón y N. Orcajada (eds.), Investigación e innovación en formación del profesorado (pp. 369-380). Murcia: Editum.

Miller, E. K., y Cohen, J. D. (2001). An integrative theory of prefrontal cortex function. Annual Review of Neuroscience, 24, 167-202.

Montiel-Nava, C., y Peña, J. A. (2001). Discrepancia entre padres y profesores en la evaluación de problemas de conducta y académicos en niños y adolescentes. Revista de Neurología, 32 (6), 506511.

Morrow, R., Wright, J., Maclure, M., Taylor, S., y Dormuth, C. (2012). Influence of relative age on diagnosis and treatment of attention-deficit/hyperactivity disorder in children. Canadian Medical Association Journal, 184, 755-762.

Muñoz Yunta, J. A., Palau, M., Salvadó, B., y Valls, A. (2006). Neurobiología del TDAH. Acta Neurológica Colombiana, 22, 184-189.

Navarro González, M. I. y García-Villamisar, D. A. (2010). El concepto de hiperactividad infantil en perspectiva: Breve análisis de su evolución histórica. Historia de la Psicología, 31 (4), 23-36.

Organización Mundial de la Salud (1995). Clasificación Estadistica Internacional de Enfermedades y Problemas Relacionados con la Salud. Décima Revisión: CIE-10. Volumen 1. Washington: Organización Panamericana de la Salud.

Orjales, I. (2001). Déficit de atención con hiperactividad manual para padres y educadores. Madrid: CEPE.

Orjales, I. (2003). Déficit de atención con hiperactividad. Diagnóstico e intervención. En E. González (ed.), Necesidades educativas específicas intervención psicoeducativa (pp. 431-467). Madrid: Editorial CCS. 
Pennington, B. F., y Ozonoff, S. (1996). Executive functions and developmental psychopathology. Journal of Child Psychology and Psychiatry and Allied Disciplines, 37, 51-73.

Pineda, D., Ardila, A., y Rosselli, M. (1999). Neuropsychological and behavioral assessment of ADHD in seven- to twelve-year old children: A discriminant analysis. Journal of Learning Disabilities, 32, 159-173.

Portellano, J. A. (1989). Fracaso escolar. Diagnóstico e intervención. Una perspectiva neurológica. Madrid: Ciencias de la Educación Preescolar y Especial.

Ramos-Loyo, J., Taracena, A. M., Sánchez-Loyo, L. M., Mature, E., y González-Garrido, A. A. (2011). Relación entre el Funcionamiento Ejecutivo en Pruebas Neuropsicológicas y en el Contexto Social en Niños con TDAH. Revista Neuropsicología, Neuropsiquiatría y Neurociencias, 11, $1,1-16$.

Rubia, K., Alegría, A., y Brinson, H. (2014). Anomalías cerebrales en el trastorno por déficit de atención/hiperactividad: una revisión. Revista de Neurología, 58 (supl. 1): S3-18.

Sandín, B. (2013). DSM-5: ¿Cambio de paradigma en la clasificación de los trastornos mentales? Revista de Psicopatología y Psicología Clínica, 18, 255-286.

Servera, M., y Cardo, E. (2007). Las ADHD Rating Scale-IV en una muestra escolar española: datos normativos y consistencia interna para maestros, padres y madres. Revista de Neurología, 45, 393-399.

Shallice, T., Marzocchi, G. M., Coser, S., Del Savio, M., Meuter, R., y Rumiati, R. (2002). Executive function profile of children with attention deficit hyperactivity disorder. Developmental Neuropshychology, 21, 43-71.

Shimamura, A. P. (2000). The role of the prefrontal cortex in dynamic filtering. Psychobiology, 28, 207-218.

Steele, R., Forehand, R., y Devine, D. (1996) Adolescent social and cognitive competence: Crossinformant and intra-individual consistency across three years. Journal of Clinical Child Psychology, 25, 60-65.

Thapar, A., Cooper, M., Jefferies, R., y Stergiakouli, E. (2012). What causes attention deficit hyperactivity disorder? Arch Dis Child, 97, 260-265.

Trujillo, A., y Díaz-Sibaja, M. A. (2007). Complicaciones en el diagnóstico del TDAH: Diferencias entre padres y profesores. Psiquiatria.com, 11 (1), 1-9.

United Nations Educations, Scintific and Cultural Organization UNESCO (2011). Las TIC accesibles y el aprendizaje personalizado para estudiantes con discapacidad: un diálogo entre los educadores, la industria, el gobierno y la sociedad civil. Sector de Comunicación e Información División de las Sociedades de Conocimiento.

Vaquerizo-Madrid, J., Estévez-Díaz, F., y Pozo-García, A. (2005). El lenguaje en el trastorno por déficit de atención con hiperactividad: competencias narrativas. Revista de Neurología, 41 (Supl. 1), S83-S89.

Verhulst, F. C., y Van der Ende, J. (1991). Four year follow up of teacher reported problem behaviors. Psichological. Medicine, 21, 965-977.

\section{Abstract}

\section{Differential patterns between parents and teachers in detecting ADHD}

INTRODUCTION. The diagnosis of Attention Deficit Disorder with Hyperactivity (ADHD) has increased in recent years. The criteria for ADHD have become clearer in the scientific community. 
However, it is a complex disorder that requires the participation of a multidisciplinary team for an appropriate diagnosis. METHOD. Taking as a sample $(\mathrm{N}=331) 6$ to 9 year-old students from Cuenca, this study analyzed the existence of differential patterns between the two ratings, using the EMA-D.D.A. Scales (García-Pérez y Magaz, 2006). Different approaches to hypothesis testing are used, and the internal consistency of the scales is valued. RESULTS. The differences observed suggest differential influence of observational context and is consistent with the results obtained in other studies. DISCUSSION. The diagnosis requires the detection at school, a clinical and a further neurological evaluation. In school screening behavioral assessment scales are used to which parents and teachers respond. The establishment of the diagnosis requires that both assessments are coincident to detect ADHD. However, studies show differences between both assessments.

Keywords: ADHD, Diagnosis, School screening, Differential analysis, Assessment of parents and teachers.

\section{Résumé}

Modèles différentiels de détection du TDAH vis-à-vis des expériences de parents et enseignants

INTRODUCTION. Le diagnostic du Trouble du Déficit de l'Attention avec Hyperactivité (TDAH) a augmenté pendant les dernières années (CDC, 2014; UNESCO, 2011: 14). La communauté scientifique a éclairé les critères sur le TDAH. Cependant, il s'agit d'un trouble complexe qui demande la participation au diagnostic d'une équipe multidisciplinaire. MÉTHODE. Dans cette étude nous analysons, en utilisant les échelles EMA-D.D.A. (García-Pérez y Magaz, 2006) et auprès un échantillon ( $\mathrm{N}=331)$ d'élèves de 6 à 9 ans provenants de Cuenca, l'existence de modèles différentiels entre les deux évaluations. Nous utilisons diverses approximations de contraste de l'hypothèse et nous analysons la consistance interne des échelles. RÉSULTATS. Les différences observées signalent l'influence différentielle du contexte observationnel, à la fois qu'elle est coïncidente avec les résultats obtenus dans d'autres études. DISCUSSION. Pour arriver au diagnostic il est nécessaire une détection scolaire, c'est-à-dire, une évaluation clinique comme neurologique. Dans la détection scolaire on utilise des échelles d'évaluation comportementale, qui sont répondues par les parents et les enseignants. Létablissement du diagnostic exige que toutes les deux évaluations soient positives, c'est-à-dire, coïncidentes sur la détection du TDAH. Les études réalisées montrent qu'il existe des différences entre les deux évaluations, fait que nous analyseront dans l'interprétation et les conclusions de ce travail.

Mots clés: TDAH, Diagnostic, Détection scolaire, Analyse différentiel, Avis des parents et des enseignants.

\section{Perfil profesional de los autores}

\section{Purificación Sánchez-Delgado (autora de contacto)}

Profesora contratada doctora en el Departamento Métodos de Investigación y Diagnóstico en Educación (MIDE), de la Facultad de F y CC. de la Educación de la Universitat de València. Es miembro 
del Grupo de Evaluación y Medición —GEM- (www.uv.es/gem) y ha impartido docencia en las áreas de Medición y Evaluación Educativas. Sus líneas de investigación giran en torno al desarrollo y validación de pruebas para medir diferentes tipos de variables educativas vinculadas al área del lenguaje, tanto orientadas para el diagnóstico individual como para la evaluación de instituciones y sistemas. Correo electrónico de contacto: purificación.sanchez@uv.es

Dirección para la correspondencia: Departamento de Métodos de Investigación y Diagnóstico en Educación, Facultad de Fa y CC. de la Educación. Avda. Blasco Ibáñez, 30. 46010 Valencia.

\section{Ana Rosa Bodoque Osma}

Profesora titular de Escuela Universitaria, del Departamento de Psicología Evolutiva y de la Educación, de la Facultad de Educación Universidad de Castilla-La Mancha (sede Cuenca. Licenciada en Psicopedagogía, imparte docencia en Logoterapia, Psicopedagogía y el Máster en Promoción de la lectura y literatura infantil. Dirige el Postgrado en Comunicador en lengua de signos (5 ediciones). Sus líneas de trabajo se centran en lenguaje y comunicación, tanto en el ámbito educativo como sanitario, así como a problemáticas específicas (como los Trastornos por Déficit de Atención) y su vinculación con el lenguaje.

Correo electrónico de contacto: rosa.bodoque@uclm.es

\section{Jesús Miguel Jornet Meliá}

Catedrático de Universidad, Departamento Métodos de Investigación y Diagnóstico en Educación (MIDE), Facultad de Fa y CC. de la Educación, Universitat de València. Catedrático de Medición y Evaluación Educativas, del área de Métodos de Investigación y Diagnóstico en Educación en la Universitat de València (España). Coordinador del GEM (www.uv.es/gem). Líneas de trabajo: diseño de instrumentos de medición y evaluación educativas (competencias y constructos socioafectivos, alumnado y profesorado), evaluación de organizaciones y sistemas educativos (dimensión educativa de la cohesión social y equidad como consecuencia de la educación).

Correo electrónico de contacto: jornet@uv.es 